\title{
Preferência Musical e Busca de Sensações Entre Jovens
}

Music Preference and Sensation Seeking Among Youngsters

Preferencia Musicale y la Búsqueda de Sensación Entre Jóvenes

Carlos Eduardo Pimentel, Valdiney Veloso Gouveia, Leconte de Lisle Coelho Júnior, Rebecca Alves Aguiar Athayde \& Tiago Jessé Souza de Lima

Universidade Federal da Paraíba
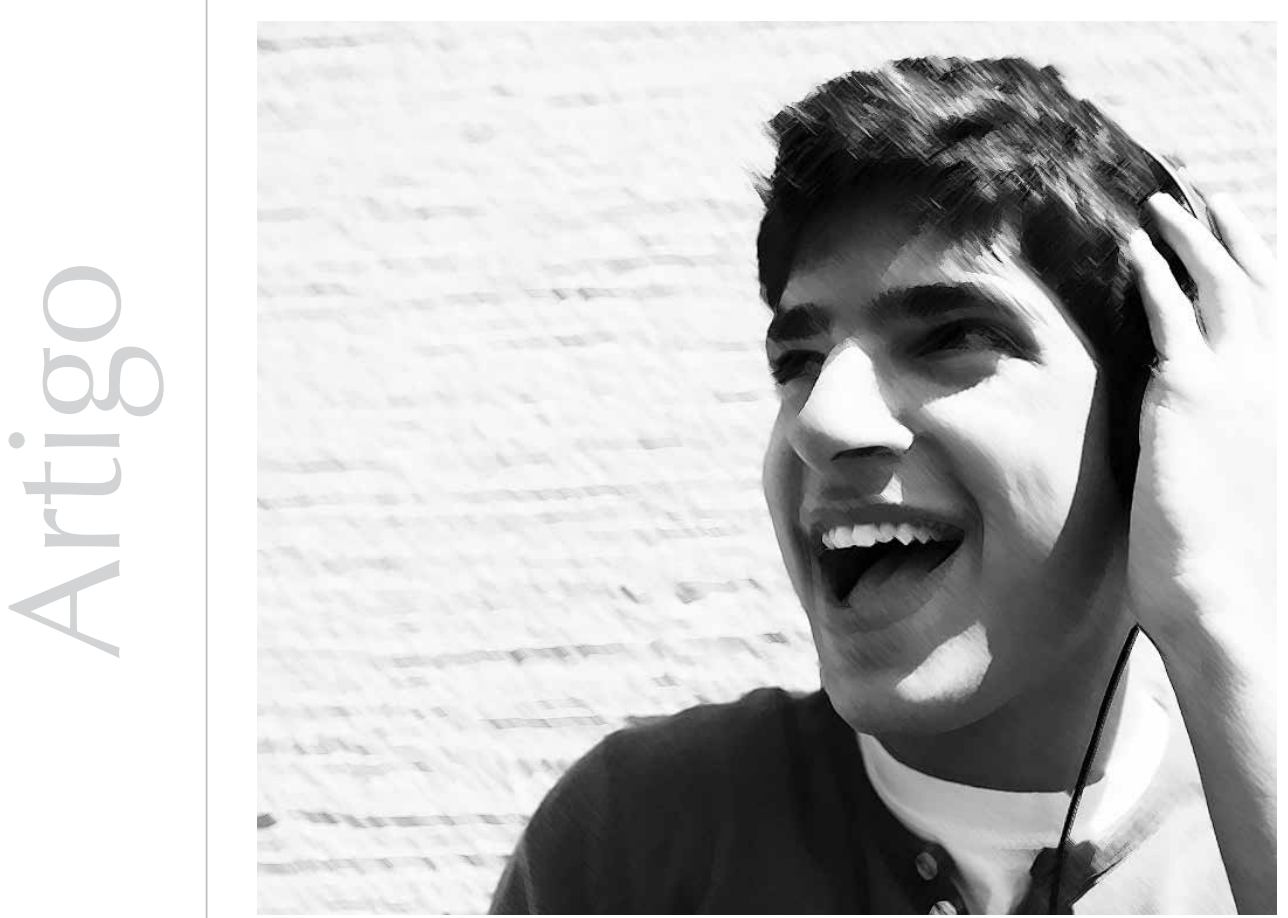
Resumo: Este estudo objetivou conhecer a correlação entre preferência musical e busca de sensações, testando modelos de mediação e moderação. Participaram 279 estudantes do ensino médio, com média de idade de 17 anos e igualmente distribuídos por sexo, que responderam a Escala de Preferência Musical, o Inventário de Arnett de Busca de Sensações e questões demográficas. Observou-se correlação da preferência por estilos musicais alternativos (positiva, como, por exemplo, rock, música eletrônica) e convencional (negativa, como, por exemplo, música clássica, música religiosa) com o fator intensidade de busca de sensações. Constatou-se também que as relações entre o fator novidade e os estilos alternativos e convencionais foram mediadas pela busca por intensidade. Testou-se a moderação da busca por novidade na relação entre intensidade e música alternativa, que não foi confirmada. Tais resultados foram discutidos com base na literatura sobre correlatos da busca de sensações, e sugere-se estudos que contribuam para entender seu efeito na preferência musical.

Palavras-chave: Preferências. Música. Percepção. Adolescentes. Sensação. Comportamento de escolha.

Abstract: This study aimed to know the correlation between music preference and sensation seeking, testing models of mediation and moderation. The participants were 279 high school students, mean age of 17 years, equally distributed by gender, who answered the Musical Preference Scale, the Arnett Inventory of Sensation Seeking and demographic questions. It was observed correlation of preferred musical styles alternative (positive, e.g., rock, electronic music) and conventional (negative, e.g., classical music, religious music) with the factor named as intensity of sensation seeking. It was also found that the correlations between novelty factor and the alternative and conventional styles were mediated by the seeking of intensity. We tested the moderation of novelty in the correlation between intensity and alternative music, which was not confirmed. These results were discussed based on the literature about the correlates of sensation seeking, suggesting studies that might contribute to understand its effects on music preference.

Keywords: Preference. Music. Perception. Adolescents. Sensation. Choice Behavior.

Reseumen: Este estudio investigó la correlación entre preferencia musical y búsqueda de sensaciones, probando modelos de mediación y moderación. Participaron 279 estudiantes de secundaria, con edad promedia de 17 años, distribuidos igualmente por género. Ellos contestaron la Escala de Preferencia Musical, el Inventario de Arnett de Búsqueda de Sensación y preguntas demográficas. Se observó correlación de la preferencia por estilos alternativo (positiva; por ejemplo, rock, música electrónica) y convencional (negativa; por ejemplo, música clásica, música religiosa) con el factor intensidad de búsqueda. También se constató que las correlaciones entre el factor novedad y los estilos alternativo y convencional fueron mediadas por búsqueda por intensidad. Se evaluó la moderación de la búsqueda por novedad en la relación entre intensidad y música alternativa, que no se confirmó. Estos hallazgos fueron discutidos según la literatura sobre los correlatos de búsqueda de sensaciones, sugieriendo estudios que contribuyan para entender su efecto en la preferencia musical.

Palabras-clave: Preferência. Música. Percepcion. Adolescentes. Sensación. Conducta de eleccion.

A preferência musical é uma variável que tem chamado a atenção de pesquisadores na Psicologia, particularmente no âmbito da pesquisa sobre traços de personalidade (Cattell \& Saunders, 1954), no qual novas pesquisas têm sido demandadas (Rentfrow, 2004; Rentfrow \& Gosling, 2003). Conceitualmente, de acordo com Boer (2009), a preferência musical pode ser compreendida como uma atitude, isto é, como uma avaliação geral em termos de favorabilidade ou de desfavorabilidade em relação a estilos musicais. Essa autora levou em conta a teoria funcional das atitudes para entender a preferência musical, descrevendo as funções avaliação de objeto, ajustamento social, defesa do ego e expressão de valores. Schwartz e Foutz (2003) sugerem que, por meio da preferência musical, a própria personalidade se expressa. De fato, a preferência musical tem estado relacionada com alguns traços ou fatores da personalidade, quer em termos dos cinco grandes (Rawlings \& Ciancarelli, 1997; Rentfrow \& Gosling, 2003; Pimentel \& Donnely, 2008) ou de outros modelos (Mccown, Keiser, Mulhearn, \& Williamson, 1997; North, Desborough, \& Skarstein, 2005; Pearson \& Dollinger, 2004).

Não é somente a traços de personalidade que a preferência está associada. As pesquisas no contexto internacional, por exemplo, 
A busca de sensações é definida por (Zuckerman 1994; Zuckerman, Bushsbaum, \& Murphy, 1980) como um traço, uma disposição que se liga a correlatos de ordem biológica, e que pode ser entendida como uma "tendência para procurar sensações e experiências novas variadas, complexas e intensas, e a disposição para correr riscos com

a finalidade de satisfazer tais experiências"

(Zuckerman, 1994,

p. 1). têm revelado correlações significativas da preferência musical com orientação política (Fox \& Williams, 1974), estilos de vida (North \& Hargreaves, 2007), valores (Boer, 2009; Leming, 1987), autoestima (Rentfrow \& Gosling, 2006), viés intergrupal (Bakagiannis \& Tarrant, 2006), risco de suicídio (Lacourse, Claes, \& Villeneuve, 2001), habilidades cognitivas (Rentfrow \& Gosling, 2003), problemas familiares, uso de drogas, comportamento sexual de risco e condutas antissociais (Arnett, 1991; Dent et al., 1992; Mulder, Bogt, Raaijmakers, \& Vollebergh, 2006; Singer, Levine, \& Jou, 1993). No âmbito dos estudos nacionais, é possível encontrar pesquisas que relacionem a preferência musical com valores e identificação grupal (Pimentel, 2004), comportamentos e atitudes antissociais (Pimentel, Gouveia, \& Vasconcelos, 2005) e com risco de suicídio (Pimentel, Gouveia, Lima, Chaves, \& Rodrigues, 2009).

Apesar do que foi anteriormente comentado, muito há ainda que pesquisar com o fim de conhecer os antecedentes e os consequentes da preferência musical. Nesse sentido, um elemento importante parece potencialmente relacionado com essa variável, sendo tratado como um traço de personalidade, ou seja, o construto busca de sensações (Arnett, 1991; Litle \& Zuckerman, 1986; McNamara \& Ballard, 1999; Weisskirch \& Murphy, 2004). Esse traço tem sido relevante para a explicação de vários comportamentos (Zuckerman, 1994), e pode contribuir igualmente para compreender a preferência musical e, inclusive, a relação desta com outras variáveis. É possível, por exemplo, que a relação entre preferência musical e comportamentos de risco seja mediada ou moderada pela busca de sensações. Demanda-se, pois, entender melhor esse construto.

\section{Busca de sensações}

A busca de sensações é definida por (Zuckerman 1994; Zuckerman, Bushsbaum, \& Murphy, 1980) como um traço, uma disposição que se liga a correlatos de ordem biológica, e que pode ser entendida como uma "tendência para procurar sensações e experiências novas variadas, complexas e intensas, e a disposição para correr riscos com a finalidade de satisfazer tais experiências" (Zuckerman, 1994, p.1). Arnett (1994), por sua vez, define esse traço como um potencial para a tomada de riscos; de acordo com sua teoria da socialização (Arnett, 1992a), os comportamentos de risco são prevalentes na adolescência devido a níveis altos de busca de sensações nessa fase da vida. Esse autor comentou que um grupo de amigos que possua níveis altos de busca de sensações muito provavelmente terá o mesmo objetivo acerca do que fazer um sábado à noite: eles se envolverão em comportamentos de risco para sua saúde e poderão colocar em risco também o bem-estar de outrem.

Arnett (1994) enfatizou, em sua definição de busca de sensações, a qualidade da estimulação sensorial. Segundo esse autor, portanto, a busca de sensações se define principalmente pela busca que envolve intensidade e novidade, estando esses fatores positivamente relacionados entre si. Em termos operacionais, comportamentos que expressam a busca por novidade expressariam o desejo de viajar para países distantes ou de ir para a Lua, por exemplo, e comportamentos relacionados com a busca por intensidade se traduziriam em escutar música em volume alto ou trabalhar melhor sob pressão, por exemplo.

Diversas pesquisas prévias têm mostrado correlações entre a busca de sensações e comportamentos sexuais arriscados, como não usar preservativo e ter vários parceiros sexuais (Arnett, 1990, 1994; Arnett \& 
Arnett (1993)

explicou que níveis altos de busca de sensações em fãs de heavy metal ocorrem em razão de esse tipo de música ser caracterizado por produzir a sensação de intensidade.
Balle-Jensen, 1993; Ledesma, Poó, \& Peltzer, 2007), uso de álcool (Andrew \& Cronin, 1997), tabaco (Carton, Jouvent, \& Widlöcher, 1994; Greene, Krcmar,Walters, Rubin, \& Hale, 2000), substâncias psicoativas (Ames, Zogg, \& Stacy, 2002; Crawford, Pentz, Chou, Li, \& Dwyer, 2003; Dubey \& Arora, 2008) e jogos de azar (McDaniel \& Zuckerman, 2003). No contexto nacional, tem sido verificada a relação de busca de sensações com valores de experimentação (Vasconcelos, 2004) e comportamentos antissociais e delitivos (Vasconcelos, Gouveia, Pimentel, \& Pessoa, 2008). Nesse sentido, o conjunto dessas pesquisas mostra que esse é um traço importante para explicar uma série de comportamentos bem como para entender construtos presumivelmente correlatos, a exemplo de traços gerais de personalidade (extroversão e abertura à mudança) e preferência musical.

\section{Preferência musical e busca de sensações}

Alguns estudos, não muitos, procuraram conhecer as relações entre preferência musical e busca de sensações. Verificou-se, por exemplo, que os altos buscadores de sensações demonstravam maior preferência por estilos de músicas anticonvencionais ou excitantes, como heavy metal, hard, soft e classic rock (Arnett, 1991, 1992b; Litle \& Zuckerman, 1986; McNamara \& Ballard, 1999). Litle e Zuckerman administraram a Escala de Busca de Sensações (SSS-Forma V) e uma medida de preferência musical em 82 estudantes estadunidenses, e comprovaram correlações positivas da pontuação total de busca de sensações com os estilos hard rock, soft rock e classic rock, e correlação negativa com trilhas sonoras. Foram verificadas também correlações positivas de fatores de busca de sensações com folk, música clássica, hard rock e solf rock, e negativas com trilhas sonoras e música religiosa. Esses autores observaram que a maioria dos jovens preferiu o estilo rock, e concluíram que esse tipo de música serve a propósitos dos buscadores de sensações no que tange à busca de estimulação sensorial.

Arnett (1991) verificou também correlações entre a preferência musical e a busca de sensações. Os principais resultados mostraram que os adolescentes que têm preferência por heavy metal apresentaram mais busca de sensações. Posteriormente, Arnett (1992b) corroborou essas relações, verificando que os adolescentes que indicaram preferência pelo hard rock e pelo heavy metal apresentaram também os mais altos níveis de busca de sensações. Congruente com sua teoria, Arnett (1993) explicou que níveis altos de busca de sensações em fãs de heavy metal ocorrem em razão de esse tipo de música ser caracterizado por produzir a sensação de intensidade.

McNamara e Ballard (1999) também verificaram relações entre preferência musical e busca de sensações entre estudantes universitários, presumivelmente jovens com maior propensão à busca de sensações. Esses pesquisadores verificaram que o rock alternativo, o rock clássico e o TOP 40 foram os estilos musicais mais preferidos pelos participantes, sendo os menos preferidos a música cristã, o heavy metal e o bluegrass, e observaram ainda que os estilos mais preferidos se correlacionaram positivamente com a busca de sensações, ao passo que os estilos menos preferidos se correlacionaram negativamente com esse fator. Esses resultados deram suporte aos achados empíricos prévios (Arnett, 1991, 1992b, 1993; Litle \& Zuckerman, 1986).

Mais recentemente, Weisskirch e Murphy (2004) procuraram conhecer a preferência musical e a busca de sensações entre jovens estudantes universitários. Esses autores 
verificaram que os jovens que tiveram pontuações mais altas nos fatores de busca de sensações denominados intensidade e novidade (e também na escala total) apresentaram maior preferência por punk e heavy metal, assim como por reggae e ska, e constataram ainda que aqueles que reportaram passar mais tempo ouvindo música punk apresentaram níveis mais altos de busca de sensações.

Em suma, considerando que a cultura exerce um papel importante na definição de traços de personalidade (Hofstede \& McCrae, 2004) e nas preferências musicais de jovens (Boer, 2009), o presente estudo busca replicar no contexto do Nordeste brasileiro os achados previamente descritos sobre a relação entre preferência musical e busca de sensações. Entretanto, busca-se ir mais além dessas pesquisas, comprovando modelos de mediação e de moderação (Baron \& Kenny, 1986) dos fatores de busca de sensações e preferência musical. Embora a preferência musical já tenha sido relacionada com outras variáveis, como com a busca de sensações, é importante conhecer a força dessa correlação em contexto brasileiro, o que possibilita reunir mais evidências empíricas que deem conta da natureza etic dessa relação.

\section{Método}

\section{Participantes}

Participaram voluntariamente desta pesquisa 279 estudantes de duas cidades do Nordeste brasileiro: João Pessoa (PB, $n=108)$ e Teresina ( $\mathrm{PI}, n=171$ ), que cursavam o ensino médio e apresentavam idades entre 13 e 38 anos $(m=17,3, d p=3,47)$; majoritariamente, eram adolescentes e jovens adultos com idades de até 28 anos (98\%), sendo a maioria de escolas públicas $(65,7 \%)$ e solteira $(65,6 \%)$, equitativamente distribuídos em relação ao sexo.

\section{Instrumentos}

Um Termo de Consentimento Livre e Esclarecido, com uma breve descrição do núcleo de pesquisa responsável e dos propósitos gerais do estudo, fazia parte da primeira folha do questionário, que continha três partes:

Escala de Preferência Musical. Com base nessa escala (Pimentel, Gouveia, \& Pessoa, 2007) e na Escala Abreviada de Preferência Musical (Gouveia, Pimentel, Santana, Chaves, \& Rodrigues, 2008; Rentfrow \& Gosling, 2003), elaborou-se um instrumento novo, com 16 itens ou estilos musicais, respondidos em escala de sete pontos, tipo Likert, que variaram de $\mathbf{1}=$ Detesto a $\mathbf{7}=$ Costo muito. De acordo com análise fatorial dos eixos principais (PAF), utilizando-se a análise paralela para decidir acerca do número de fatores a extrair, sem fixar rotação, identificaram-se três fatores (que explicaram conjuntamente $37,2 \%$ da variância total): música alternativa ( $\alpha=0,68$; heavy metal, rap, funk, dance/música eletrônica, pop music, punk, reggae), música de massa ( $\alpha$ $=0,78$; pagode, forró, samba e sertaneja) e música convencional ( $\alpha=0,47$; MPB, trilhas sonoras, música religiosa e música clássica).

Inventário de Busca de Sensações de Arnett (AISS; Arnett, 1994). Utilizou-se uma versão abreviada dessa escala, proposta por Gouveia et al. (2010), que era formada por 12 itens $(\alpha=0,56)$ distribuídos em dois fatores: novidade ( $\alpha=0,42$; por exemplo, "Eu gostaria de viajar para lugares que são estranhos e distantes") e intensidade ( $\alpha=$ 0,51; "Eu gosto de filmes em que há muitas explosões e perseguições de carros"). Essa estrutura bifatorial recebeu apoio empírico por meio de análise fatorial confirmatória: $\chi^{2}$ / g.l. $=1,82, \mathrm{GFI}=0,95, \mathrm{AGFI}=0,92$, RMSEA $=0,054($ IC 90\% $=0,037-0,071)$, PCLOSE $=0,326$. Cada item é respondido em escala de tipo Likert, de quatro pontos, 
variando de $\mathbf{1}=$ Descreve-me muito bem a $\mathbf{4}=$ Não me descreve totalmente. Quanto maior a pontuação, mais busca de sensações a pessoa apresenta.

Questionário demográfico. Na última página do questionário, constavam perguntas de natureza demográfica (cidade, escola, idade e sexo) que tinham a finalidade de caracterizar melhor os participantes do estudo.

\section{Procedimento}

Inicialmente, contataram-se os diretores de escolas públicas e privadas do ensino médio de João Pessoa e Teresina. Após seu consentimento, os questionários foram aplicados de modo coletivo em sala de aula, mas as respostas foram individuais. Dois bolsistas de Iniciação Científica do curso de Psicologia de uma universidade pública na Paraíba e uma psicóloga colaboradora da pesquisa no Piauí foram responsáveis pela coleta de dados. Estes se identificavam como participantes de uma equipe de pesquisa interessada em saber como os jovens pensam e se comportam no cotidiano. Os aspectos éticos para se realizar a pesquisa com seres humanos foram salvaguardados, o que incluiu a participação voluntária e anônima, o direito ao sigilo e a informação de que a pesquisa não oferecia risco ao respondente. Todos foram informados que poderiam interromper sua participação na pesquisa a qualquer momento, sem que isso acarretasse prejuízo, e que os resultados da pesquisa seriam usados unicamente com fins científicos. Em média, 20 minutos foram necessários para realizar essa atividade.

\section{Análise dos dados}

A digitação e a análise dos dados foram realizadas no SPSS 15, e incluíram análises descritivas (média, desvio-padrão, percentuais) e inferenciais. Esse programa permitiu obter correlações de Pearson e análise multivariada de variância (MANOVA). O AMOS 7 foi empregado para testar os modelos de mediação (Kenny, 2010a), tendo sido considerados os seguintes indicadores de ajuste (Byrne, 2001; Garson, 2003):

Razão $\chi^{2}$ / g.l. O qui-quadrado do modelo é dividido por seus graus de liberdade, recomendando-se como indicação de adequação do modelo valores entre 2 e 3 (ou menos), mas podem ser aceitos até 5 .

GFI e CFI. Os Goodness-of-Fit Index (GFI) e Comparative-Fit Index (CFI) são índices que falam do ajuste do modelo, devendose se situar próximos de 1, acima de 0,90 preferencialmente.

RMSEA. Este índice, Root-Mean-Square Error of Approximation, deve ser mais próximo de zero para considerar o ajuste adequado; o ponto de corte é 0,05 ou menos, mas são aceitos valores de 0,10.

ECVI, CAIC e Teste de Diferença entre Quiquadrados $\left(\Delta \chi^{2}\right)$. O Expected Cross-Validation Index (ECVI) e o Consistent Akaike Information Criterion (CAIC) são utilizados para comparar modelos rivais. O modelo com menores valores de ECVI e CAIC pode ser considerado o mais adequado. Quanto ao $\Delta \chi^{2}$, se for significativo, indica melhor ajuste do modelo com menor valor de $\chi^{2}$.

\section{Resultados}

\section{Estatísticas descritivas}

Inicialmente, calcularam-se estatísticas descritivas para cada estilo musical e os fatores de busca de sensações. Os estilos musicais preferidos pelos jovens foram: forró ( $m=5,24, d p=2,09)$ e dancel música eletrônica ( $m=5,14, d p=2,09$ ). Considerando o ponto central da escala de resposta (4 = Tanto faz), os seguintes estilos são medianamente preferidos: trilhas sonoras ( $m=4,62, d p=1,19)$, pop music $(m=4,47$, $d p=2,01)$, pagode $(m=4,36, d p=2,21)$, 
música religiosa ( $m=4,22, d p=2,12)$, rock $(m=4,08, d p=2,26)$, funk $(m=4,03, d p$ $=2,12)$ e samba $(m=4,02, d p=2,08)$, e, finalmente, eles parecem preferir menos os seguintes: MPB $(m=3,86, d p=1,90)$, reggae $(m=3,72, d p=2,13), \operatorname{rap}(m=3,45, d p$ $=2,20)$, sertaneja $(m=3,42, d p=1,98)$, música clássica $(m=2,94, d p=1,95)$, punk ( $m=2,76, d p=1,94)$ e heavy metal $(m=$ $2,70, d p=1,98)$. Quanto às pontuações nos fatores de busca de sensações, observaramse as seguintes médias: novidade $(m=2,53$, $d p=0,57)$, intensidade $(m=2,52 ; d p=$ $0,55)$ e pontuação total $(m=2,53 ; d p=$ $0,44)$. Considerando a mediana da escala de resposta, situada entre $\mathbf{2}$ (Não me descreve muito bem) e $\mathbf{3}$ (Descreve-me um pouco), percebe-se que os respondentes se situaram próximo ao centro da distribuição.

Em suma, os jovens participantes desta pesquisa gostam mais de forró e menos de heavy metal. Isso sugere que apresentam pontuações não tão altas em busca de sensações, o que foi observado, portanto, são jovens que cumprem padrões normativos e apreciam estilos musicais mais de massa, com menos probabilidade de se envolverem em comportamentos de busca de sensações.

\section{Correlações entre fatores de busca de sensações e preferências musicais}

A pontuação total de busca de sensações apresentou correlação positiva com a preferência pelos seguintes estilos musicais: rock $(r=0,24, p<0,001)$, pop music $(r=$ $0,19, p<0,01)$, rap $(r=0,19, p<0,01)$, dance/música eletrônica $(r=0,15, p<$ $0,05)$ e reggae $(r=0,12, p<0,05)$, e o fez negativamente com a preferência pelos estilos de música religiosa $(r=-0,19, p<0,01)$ e música sertaneja $(r=-0,14, p<0,05)$. Quanto aos fatores específicos de busca de sensações, suas correlações com a preferência pelos estilos foram os seguintes: (1) intensidade se correlacionou com rap $(r=0,27, p<0,001)$, música religiosa $(r=-0,24, p<0,001)$, pop music $(r=0,21, p<0,001)$, música sertaneja $(r$ $=-0,18, p<0,01)$, música clássica $(r=-0,15$, $p<0,05)$, dance/música eletrônica $(r=0,14$, $p<0,05)$, reggae $(r=0,14, p<0,05)$ e funk $(r=0,13, p<0,05)$, e novidade somente o fez com rock $(r=0,17, p<0,05)$.

Tendo em conta os fatores de preferência musical, verificaram-se as seguintes médias: música de massa $(m=4,26, d p=1,62)$, música convencional $(m=3,91, d p=$ $1,22)$ e música alternativa $(m=3,78, d p=$ 1,15). A música alternativa se correlacionou positivamente com o fator intensidade $(r=$ $0,29, p<0,001)$ e com a pontuação total do AISS ( $r=0,28 ; p<0,001)$; em direção oposta, observou-se que o fator intensidade se correlacionou negativamente com música convencional $(r=-0,20, p<0,01)$.

\section{Análises de mediação e moderação}

Antes de proceder à análise de mediação com a amostra total, por meio de uma MANOVA, verificou-se não haver diferenças nos fatores de preferência musical e busca de sensações em relação às cidades João Pessoa e Teresina (Lambda de Wilks $=0,957, F=2,243, p$ $>0,05)$, portanto, procedeu-se ao teste de análise de mediação com o conjunto total de participantes, uma vez que o tamanho da amostra é uma característica importante para essa estatística, sendo recomendados ao menos 200 sujeitos (DiStefano \& Hess, 2005; Weston \& Gore, 2006). Essa análise procurou saber se a busca de sensações prediz a preferência musical, testando modelos de mediação. Inicialmente, verificou-se esse modelo tendo como variáveis antecedentes os fatores de busca por novidade e intensidade (correlacionados entre si) e consequentes a preferência por músicas dos tipos alternativa e convencional. 
Considerando os modelos de efeitos diretos, o Modelo 2 com os fatores de busca de sensações correlacionados entre si apresentou melhores índices de ajuste aos dados do que o Modelo 1 , com o qual tais fatores não estavam correlacionados. Na Figura 1, são apresentadas as relações entre as variáveis do Modelo 2, que apresentou pesos de regressão estatisticamente diferentes de zero $(p<0,001)$, com exceção da predição da música alternativa pelo fator de busca de sensações denominado novidade.

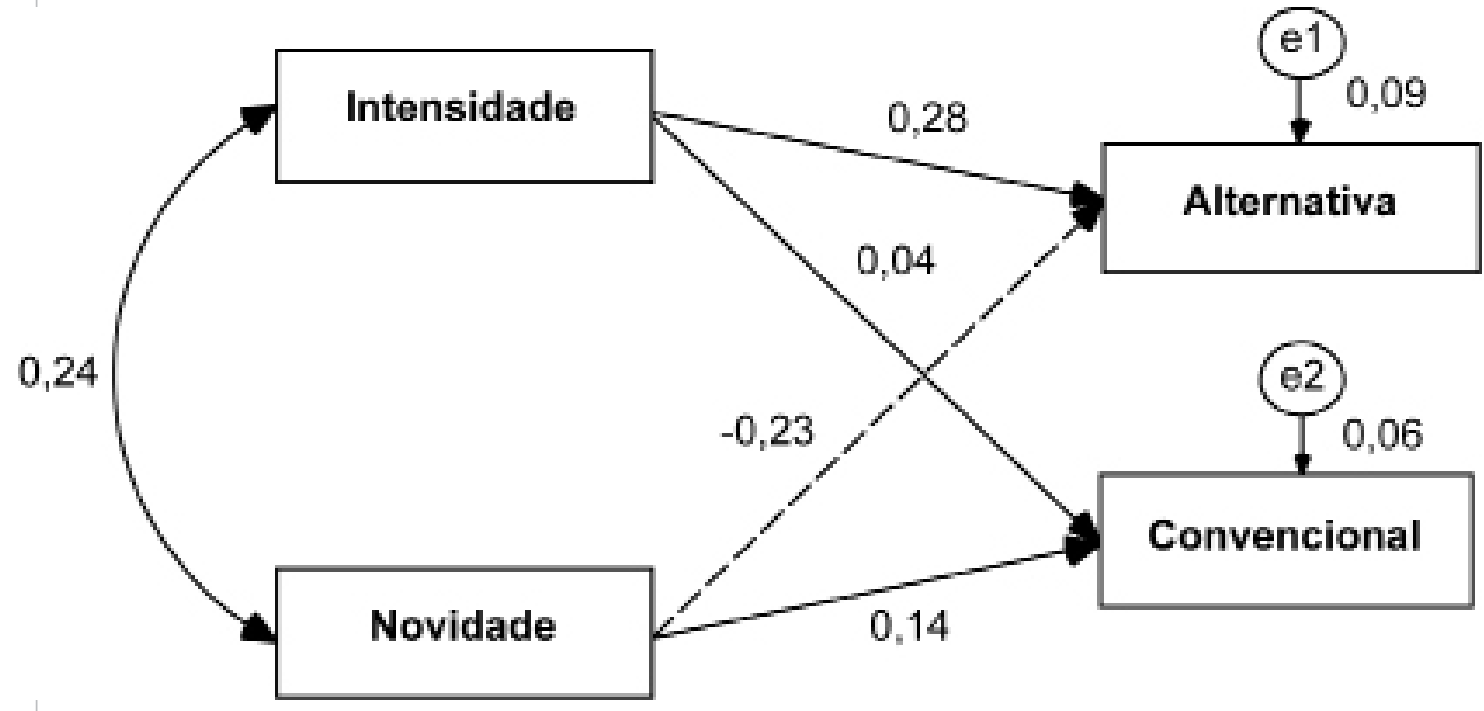

Figura 1. Modelo de efeitos diretos

Com o propósito de verificar os efeitos indiretos, testaram-se quatro modelos por meio de bootstrap (reamostragem) paramétrico, com o método bias-correct confidence intervals (Cheung \& Lau, 2008), que tem sido recomendado para testes desse tipo de análise (Hayes, 2010; Kenny, 2010a). Utilizaram-se 2.000 amostras simuladas e intervalo de confiança de $90 \%$ para se verificar os efeitos diretos e indiretos. Os resultados principais são mostrados na Tabela 1 (Modelos 3 a 6).

Tabela 1. Índices de ajuste dos modelos diretos e indiretos de busca de sensações e preferência musical

$\begin{array}{llllllllll}\text { Modelos } & \mathrm{c}^{2} & \text { g.I. } & \text { GFI } & \text { CFI } & \text { RMSEA (IC90\%) } & \text { rc }^{2} & \text { rg.I } & \text { p } & \text { CAIC }\end{array}$

ECVI

Modelo de efeitos diretos

$\begin{array}{cccccc}\text { Modelo } 1 & 16,369 & 2 & 0,97 & 0,72 & 0,161(0,095 ; 0,237) \\ \text { Modelo } 2 & 0,009 & 1 & 1 & 1 & 0,000(0,000 ; 0,056)\end{array}$

$69,419 \quad 0,116$

Modelo $2 \quad 0,009$

Modelos de mediação

Modelo $3 \quad 31,149$

$\begin{array}{lll}3 & 0,94 & 0,36\end{array}$

$0,199(0,144 ; 0,260)$

$59,690 \quad 0,065$

Modelo 4

$\begin{array}{llll}5,600 & 3 & 0,99 & 0,95\end{array}$

$0,056(0,000 ; 0,127)$

25,5490

$82,567 \quad 0,180$

Modelo 5

0,419

$2 \quad 1 \quad 1$

$0,000(0,000 ; 0,073)$

$5,18110,02$

$52,019 \quad 0,071$

Modelo 6

0,009

$1 \quad 1 \quad 1$

$0,000(0,000 ; 0,056)$

$\begin{array}{lll}0,41 & 1 & 0,52\end{array}$

$53,469 \quad 0,059$

$59,690 \quad 0,065$ 
Nota. Modelo 1 = modelo de efeitos diretos de intensidade e novidade em alternativa e convencional; Modelo $2=$ modelo de efeitos diretos de intensidade e novidade (não correlacionados) em alternativa e convencional; Modelo 3 $=$ modelo de efeitos indiretos da intensidade em alternativa e convencional; Modelo $4=$ modelo de efeitos indiretos da novidade em alternativa e convencional (mediação completa); Modelo $5=$ modelo de efeitos indiretos e diretos da novidade em alternativa e convencional (mediação parcial); Modelo 6 = modelo de efeitos indiretos novidade em alternativa e convencional e indiretos da novidade em convencional (mediação parcial).

De acordo com essa tabela, embora não tenha apresentado os melhores indicadores de ajuste, o Modelo 4, que indica mediação completa da preferência musical pela busca de sensações, apresentou valores aceitáveis, com todos os pesos de regressão estatisticamente diferentes de zero $(p<0,001)$. Destaca-se que todas as relações se deram na direção esperada. Além de efeitos diretos de intensidade nos fatores de preferência musical, comprovaram-se também efeitos indiretos padronizados de novidade em música convencional (IC 90\% $=-0,18 ;-0,05, p$ $<0,001$ ) e em música alternativa (IC $90 \%=0,08 ; 0,23, p<0,001)$. Isso sugere que, quanto mais os jovens buscam novidade e intensidade, mais preferem música alternativa e menos música convencional. O conjunto dessas relações é retratado na Figura 2.

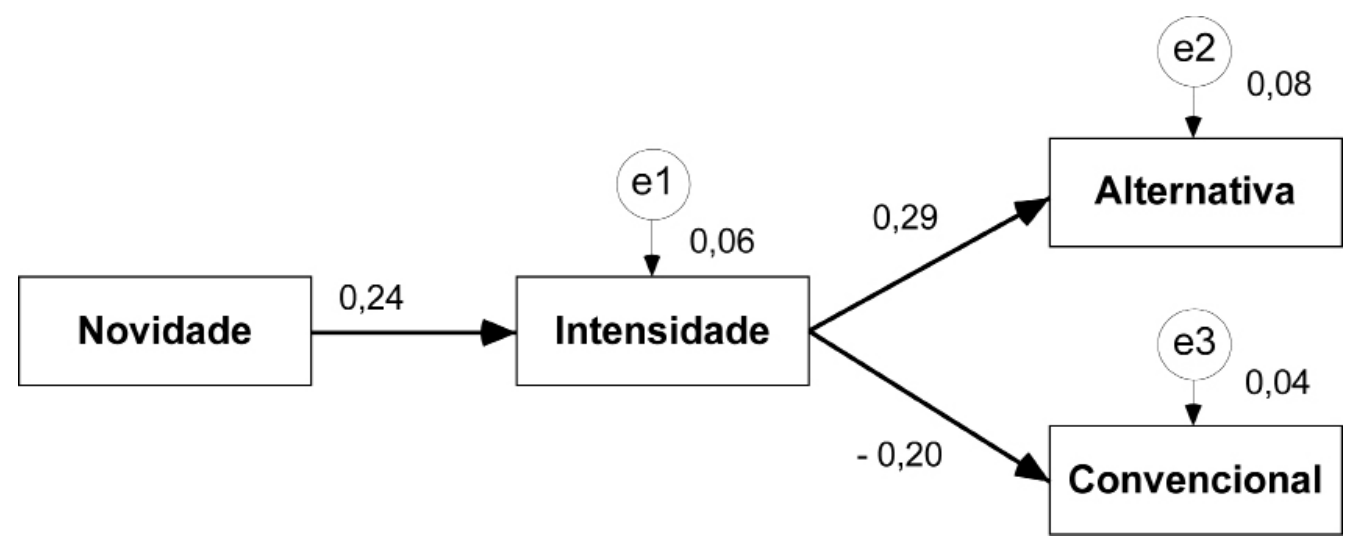

Figura 2. Modelo de mediação completa

Os Modelos 5 e 6 mostraram que o fator novidade predisse direta e positivamente a preferência por música convencional. Mesmo que esses modelos, no geral, tenham apresentado índices melhores do que o anteriormente descrito, a relação previamente indicada não era esperada, portanto, recomenda-se tratá-los com alguma cautela.

Por fim, buscou-se verificar se o fator novidade moderaria as relações entre intensidade e música alternativa, seguindo-se o procedimento recomendado na literatura especializada (Miles \& Shevlin, 2001; Kenny, 2010b). Inicialmente, verificou-se a predição dos fatores intensidade (zIntensidade) e novidade (zNovidade) na música alternativa, que apresentaram pesos de regressão padronizados de 0,28 e 0,04, respectivamente. Entretanto, a moderação não pôde ser confirmada, pois a entrada do termo de interação (variável moderadora [zNovidade] x variável independente [zIntensidade]) não mudou tais relações, permanecendo as direções e as magnitudes previamente descritas.

\section{Discussão}

Na presente pesquisa, procurou-se conhecer as correlações entre a preferência musical e a busca de sensações entre adolescentes e jovens adultos. Nesse contexto, o fator intensidade foi 
mais preponderante. Porém, além de tais correlações, comprovaram-se ainda padrões de mediação, considerando os fatores de busca por novidade e intensidade e os de música alternativa e música convencional. Tratados em conjunto, esses resultados corroboram a teoria psicológica de busca de sensações (Litle \& Zuckerman, 1983; Zuckerman, 1994), especificamente no que se refere à busca de intensidade na estimulação sensorial (Arnett, 1994). Destacase ainda que achados previamente descritos apoiam pesquisas que descrevem correlações entre a preferência por estilos musicais considerados excitantes (por exemplo, rap, rock) e a busca por sensações (Arnett, 1991, 1992b, 1993; Litle \& Zuckerman, 1983; McNamara \& Ballard, 1999; Weisskirch \& Murphy, 2004).

Uma contribuição importante da presente pesquisa diz respeito às correlações da dance music com fatores de busca de sensações. Por exemplo, a preferência por esse estilo musical se relacionou positivamente com o fator intensidade com o fator geral de busca de sensações. De modo semelhante, intensidade se correlacionou com a preferência por pop music, rock e funk, sendo que o rock o fez também com o fator novidade. No estudo de Weisskirch e Murphy (2004), que também usaram o AISS, o heavy metal foi o estilo que se correlacionou com intensidade, novidade e a pontuação total de busca de sensações. Por outro lado, constatou-se uma correlação negativa entre a preferência por música religiosa e o fator intensidade. Isso poderia ser esperado, de acordo com o que se presume na literatura (Singer, Levine, \& Jou, 1993; Zuckerman, 1994).

\section{Análises de mediação}

Quanto às análises de mediação, utilizou-se procedimento recentemente recomendado na literatura (Cheung \& Lau, 2008; Hayes, 2010; Kenny, 2010a). Verificou-se que a relação entre busca por novidade e preferência por música alternativa e música convencional é mediada (mediação completa, isto é, Modelo 4) pela busca por intensidade. Esse modelo foi testado com diversos modelos alternativos, como tem sido sugerido (DiStefano \& Hess, 2005; Weston \& Gore, 2006). Nesse sentido, Arnett (1993) explicou que a preferência por heavy metal leva à busca de intensidade na estimulação sensorial, o que é corroborado na presente pesquisa com a inclusão de outros estilos musicais excitantes em um fator chamado de música alternativa. Segundo Arnett (1994), o fator intensidade pode expressar-se em comportamentos relacionados com a música, como escutá-la em volume alto, o que é comum quando se trata de estilos alternativos.

O Modelo 2, em que a busca de sensações predisse diretamente a preferência por estilos musicais alternativos e convencionais, apresentou índices de ajuste satisfatórios, e, nesse sentido, pode ser considerado equivalente ao Modelo 4 no que diz respeito a sua plausibilidade (McDonald \& Ro, 2002), porém a virtude daquele reside nas relações de mediação.

Considerando o fator música alternativa e sua relação com intensidade, possivelmente se fosse observada preferência alta por músicas desse estilo teria sido verificado efeito direto maior de busca de sensações. Nesse ponto, destaca-se que o efeito direto da busca por novidade na música convencional não era esperado, pois deu-se em direção contrária ao observado nas correlações, aspecto que demandará estudos futuros.

\section{Limitações da pesquisa}

Apesar de cumpridos os objetivos desta pesquisa, é necessário pensar acerca de suas 
limitações potenciais. No caso, contar com amostra não aleatória sugere restrição quanto à generalização dos resultados, contudo, pondere-se que os achados descritos são consoantes com pesquisas prévias. Outro aspecto importante é que não foi possível verificar propriamente preferência (no sentido de favorabilidade ou de atitudes positivas) com relação a alguns estilos musicais, como o heavy metal, estilo que tem sido relacionado com a busca de sensações, sendo que, no presente estudo, verificouse que se trata do estilo menos preferido pelos adolescentes e jovens adultos, talvez indicando a natureza emic da preferência musical, entendendo-se essa terminologia a especificidade cultural e temporal dessa preferência. A propósito, no estudo de Litle e Zuckerman (1983), realizado no contexto estadunidense, o estilo musical preferido foi o rock, assim como ocorreu naquele descrito por McNamara e Ballard (1999). No estudo de Weisskirch e Murphy (2004), porém,o reggae foi o preferido, enquanto Arnett (1993, 1991, 1992b) identificou número expressivo de fãs de heavy metal. No presente estudo, entretanto, preferiu-se o forró, que é típico do Nordeste, mas que vem ganhando destaque no cenário nacional.

\section{Considerações finais}

De acordo com o levantamento realizado, esta é a primeira pesquisa em país de língua portuguesa que verifica a relação entre preferência musical e busca de sensações. Os resultados foram coerentes na maioria das relações encontradas. Espera-se que este estudo instigue replicações no contexto em que esta pesquisa se desenvolveu e em outras regiões do País. É importante que se verifique essa relação em fatores e níveis distintos de busca de sensações, considerando grupos que valorizam tanto estilos musicais alternativos como convencionais. A avaliação de possíveis moderadores e mediadores (Baron \& Kenny, 1986) dessa relação, como a religiosidade (Arnett, 1998) e o sexo (Roberti, 2004) dos participantes, deve ser incentivada. Do mesmo modo, devem-se considerar outros traços de personalidade, diferentes idades e fases do desenvolvimento, visando a contribuir para a explicação da preferência musical (Rentfrow \& Gosling, 2003). 


\section{Carlos Eduardo Pimentel}

Doutor em Psicologia Social, do Trabalho e das Organizações pela Universidade de Brasília e Professor Adjunto do Departamento de Psicologia da Universidade Federal da Paraíba, João Pessoa - PB - Brasil.

E-mail: carlosepimentel@bol.com.br

\section{Valdiney Veloso Gouveia}

Doutor em Psicologia Social pela Universidade Complutense de Madri e Professor Titular da Universidade Federal da Paraíba, João Pessoa - PB - Brasil.

E-mail: vvgouveia@gmail.com

\section{Leconte de Lisle Coelho Júnior}

Doutor em Psicologia pela Universidade Federal do Espírito Santo e Professor da Faculdade Maurício de Nassau, Campina Grande - PB - Brasil.

E-mail: lecontey@yahoo.com.br

\section{Rebecca Alves Aguiar Athayde}

Doutoranda em Psicologia Social na Universidade Federal da Paraíba, João Pessoa - PB - Brasil.

E-mail: bekinhacfa@hotmail.com

\section{Tiago Jessé Souza de Lima}

Doutorando em Psicologia Social na Universidade Federal da Paraíba, João Pessoa - PB - Brasil.

E-mail: tiago.souzalima@hotmail.com

Endereço para envio de correspondência:

Universidade Federal da Paraíba, Centro de Ciências Humanas Letras e Artes, Campus I, Departamento de Psicologia, Cidade Universitária, Castelo Branco. CEP: 58051-900. João Pessoa, PB. 


\section{Referências}

Ames, S. L., Zogg, J. B., \& Stacy, A. W. (2002). Implicit cognition, sensation seeking, marijuana use and driving behavior among drug offenders. Personality and Individual Differences, 33(7), 1055-1072. doi: http://dx.doi.org/10.1016/S01918869(01)00212-4

Andrew, M., \& Cronin, C. (1997). Two measures of sensation seeking as predictors of alcohol use among high school males. Personality and Individual Differences, 22(3), 393-401.

Arnett, J. (1990). Contraceptive use, sensation seeking, and adolescent egocentrism. Journal of Youth and Adolescence, 19(2), 171-180. doi: 10.1007/BF01538720

Arnett, J. (1991). Heavy metal music and reckless behavior among adolescents. Journal of Youth and Adolescence, 20(6), 573-592. doi: 10.1007/BF01537363

Arnett, J. (1992a). Socialization and adolescent reckless behavior: A reply to Jessor. Developmental Review, 12(4), 391-409.

Arnett, J. (1992b). The soundtrack of recklessness: Music preference and reckless behavior among adolescents. Journal of Youth and Adolescence, 7(3), 313-331. doi: 10.1177/074355489273003

Arnett, J. (1993). Three profiles of heavy metal fans: A taste for sensation and a subculture of alienation. Qualitative Sociology, 16(4), 423-443. doi: 10.1007/BF00989973

Arnett, J. (1994). Sensation seeking: A new conceptualization and a new scale. Personality and Individual Differences, 16(2), 289-296.

Arnett, J. (1998). Risk behaviors and family role transitions during the twenties. Journal of Youth and Adolescence, 27(3), 301-320.

Arnett, J. J., \& Balle-Jensen, L. (1993). Cultural bases of risk behavior: Danish adolescents. Child Development, 64(6), 1842-1855.

Bakagiannis, S., \& Tarrant, M. (2006). Can music bring people together? Effects of shared musical preference on intergroup bias in adolescence. Scandinavian Journal of Psychology, 47(2), 129-136. doi: 10.1111/j.1467-9450.2006.00500.x

Baron, R. M., \& Kenny, D. A. (1986). The moderator-mediator variable distinction in social psychological research: Conceptual, strategic and statistical considerations. Journal of Personality and Social Psychology, 51(6), 1173-1182. doi: 10.1037/00223514.51.6.1173

Boer, D. (2009). Music makes the people come together: Social functions of music listening for young people across cultures. Unpublished doctoral dissertation, Victoria University of Wellington, New Zealand.

Byrne, B. M. (2001). Structural equation modeling with AMOS: Basic concepts, applications, and programming. Mahwah: Lawrence Erlbaum Associates.

Carton, S., Jouvent, R., \& Widlöcher, D. (1994). Sensation seeking, nicotine dependence, and smoking motivation in female and male smokers. Addictive Behavior, 19(3), 219-227. doi: http:// dx.doi.org/10.1016/0306-4603(94)90026-4

Cattell, R. B., \& Saunders, D. R. (1954). Musical preferences and personality diagnosis: A factorization of one hundred and twenty themes. Journal of Social Psychology, 39, 3-24.
Cheung, G. W., \& Lau, R. S. (2008). Testing mediation and suppression effects of latent variables: Bootstrapping with structural equation models. Organization Research Methods, 11(2), 296-325. doi: 10.1177/1094428107300343

Crawford, A. M., Pentz, M. A., Chou, C-P., Li, C., \& Dwyer, J. H. (2003). Parallel developmental trajectories of sensation seeking and regular substance use in adolescents. Psychology of Addictive Behaviors, 17(3), 179-192. doi: 10.1037/0893-164X.17.3.179

Dent, C. W., Galaif, J., Sussman, S., Stacy, A. W., Burton, D., \& Flay, B. R. (1992). Music preference as a diagnostic indicator of adolescent drug use. American Journal of Public Health, 82(1), 124.

DiStefano, C., \& Hess, B. (2005). Using confirmatory factor analysis for construct validation. Journal of Psychoeducational Assessment, 23(3), 225-241.

Dubey, C., \& Arora, M. (2008). Sensation seeking level and drug of choice. Journal of the Indian Academy of Applied Psychology, 34(1), 73-82.

Fox, W.S. \& Williams, J.D. (1974). Political orientation and music preferences among college students source. The Public Opinion Quarterly, 38(3), 352-371.

Garson, G. D. (2003). PA 765 Statnotes: An online textbook. Recuperado em 17 maio, 2005, de: http://www2.chass.ncsu. edu/garson/pa765/statnote.htm

Gouveia, V. V., Pimentel, C. E., Santana, N. L., Chaves, W. A., \& Rodrigues, C. A. (2008). Escala abreviada de preferência musical (STOMP): evidências de sua validade fatorial e consistência interna. Psico-PUCRS, 39(2), 201-210.

Gouveia, V. V., Pimentel, C. E., Gouveia, R. S. V., Freires, L. A., Athayde, R. A. A., Araújo, R. C. R. (2010). Inventário de Arnett de busca de sensações (AISS): testando diferentes modelos fatoriais. Psico-USF, 15(2), 181-191. doi: http://dx.doi.org/10.1590/S141382712010000200006

Greene, K., Krcmar, M., Walters, L. H., Rubin, D. L., \& Hale, L. (2000). Targeting adolescent risk-taking behaviors: The contributions of egocentrism and sensation-seeking. Journal of Adolescence, 23(4), 439-461. doi: http://dx.doi.org/10.1006/ jado. 2000.0330

Hayes, A. (2010). Facebook group statistical mediation analysis. Discussion board. Recuperado em 18 mar., 2010, de http://www. facebook.com/home.php\#/group.php?gid=44574520333

Hofstede, G., \& McCrae, R. R. (2004). Personality and culture revisited: Linking traits and dimensions of culture. Cross-Cultural Research, 38(1), 52-88. doi: 10.1177/1069397103259443

Kenny, D. A. (2010a). Mediation. Recuperado em 24 abr., 2010, de http://davidakenny.net/cm/mediate.htm

Kenny, D. A. (2010b). Moderation. Recuperado em 06 maio, 2010, de:http://davidakenny.net/cm/moderation.htm

Lacourse, E., Claes, M., \& Villeneuve, M. (2001). Heavy metal music and adolescent suicidal risk. Journal of Youth and Adolescence, 30(3), 321-331. doi: 10.1023/A:1010492128537

Ledesma, R., Poó, F., \& Peltzer, R. (2007). Búsqueda impulsiva de sensaciones y comportamiento de riesgo en la conducción. Avaliação Psicológica, 6(2), 117-125. 
Leming, J. S. (1987). Rock music and the socialization of moral values in early adolescents. Youth \& Society, 18(4), 363-383. Recuperado em 17 de maio de 2005 de https://www.ncjrs.gov/ App/Publications/abstract.aspx?!D =106614

Litle, P., \& Zuckerman, M. (1986). Sensation seeking and music preferences. Personality and Individual Differences, 7(4), 575578. doi: http://dx.doi.org/10.1016/0191-8869(86)90136-4

McCown, W., Keiser, R., Mulhearn, S., \& Williamson, D. (1997). The role of personality and gender in preferences for exaggerated bass in music. Personality and Individual Differences, 23(4), 543547. doi: http://dx.doi.org/10.1016/S0191-8869(97)00085-8

McDaniel, S. R., \& Zuckerman, M. (2003). The relationship of impulsive sensation seeking and gender to interest and participation in gambling activities. Personality and Individual Differences, 35(6),1385-1400. doi: http://dx.doi.org/10.1016/ S0191-8869(02)00357-4

McDonald, R. P., \& Ho, M.-H. R. (2002). Principles and practice in reporting structural equation analyses. Psychological Methods, 7(1), 64-82. doi:10.1037/1082-989X.7.1.64

McNamara, L., \& Ballard, M. E. (1999). Resting arousal, sensation seeking, and music preference. Genetic, Social, and General Psychology Monographs, 125(3), 229-250.

Miles, J., \& Shevlin, M. (2001). Applying regression and correlation: A guide for students and researchers. London: Sage Publications.

Mulder, J., Bogt, T., Raaijmakers, Q., \& Vollebergh, W. (2006). Music taste groups and problem behavior. Journal of Youth and Adolescence, 36(3), 313-324. doi: 10.1007/s10964-006-9090-1

North, A. C., \& Hargreaves, D. J. (2007). Lifestyle correlates of musical preference: 3 . travel, money, education, employment and health. Psychology of Music, 35(3), 473-497. doi: $10.1177 / 0305735607072656$

North, A. C., Desborough, L., \& Skarstein, L. (2005). Musical preference, deviance, and attitudes towards music celebrities. Personality and Individual Differences, 38(8), 1903-1914. doi: http://dx.doi.org/10.1016/j.paid.2004.11.016

Pearson, J. L., \& Dollinger, S. J. (2004). Music preference correlates of jungian types. Personality and Individual Differences, 36(1), 10051008. doi: http://dx.doi.org/10.1016/S0191-8869(03)00168-5

Pimentel, C. E. (2004). Valores humanos, preferência musical, identificação grupal e comportamentos anti-sociais. Dissertação de mestrado. Universidade Federal da Paraíba, Paraíba, PB.

Pimentel, C. E., \& Donnelly, E. D. O. P. (2008). A relação da preferência musical com os cinco grandes fatores da personalidade. Psicologia: Ciência e Profissão, 28(4), 696-713. doi: http://dx.doi.org/10.1590/S1414-98932008000400004

Pimentel, C. E., Gouveia, V. V., \& Vasconcelos, T. C. (2005). Preferência musical, atitudes e comportamentos anti-sociais entre estudantes adolescentes: Um estudo correlacional. Estudos de Psicologia, 22(4), 403-413. doi: http://dx.doi.org/10.1590/ S0103-166X2005000400008

Pimentel, C. E., Gouveia, V. V., \& Pessoa, V. S. (2007). Escala de preferência musical: construção e comprovação da sua estrutura fatorial. Psico-USF, 12(2), 145-155.
Pimentel, C. E., Gouveia, V. V., Lima, N., Chaves, W. A., \& Rodrigues, C. A. (2009). Preferência musical e risco de suicídio entre jovens. Jornal Brasileiro de Psiquiatria, 58(1), 26-33.

Rawlings, D., \& Ciancarelli, V. (1997). Music preference and the five-factor model of the NEO Personality Inventory. Psychology of Music, 25(2), 120-132. doi: 10.1177/0305735697252003

Rentfrow, P. J. (2004). Message in a ballad: Personality judgements based on music preferences. Dissertation of Doctor of Philosophy, Faculty of the Graduate School at University of Texas, Austin.

Rentfrow, P. J., \& Gosling, S. D. (2003). The do re mi's of everyday life: The structure and personality correlates of music preference. Journal of Personality and Social Psychology, 84(6), 226-236. doi: 10.1037/0022-3514.84.6.1236

Rentfrow, P. J., \& Gosling, S. D. (2006). Message in a ballad: The role of music preferences in interpersonal perception. Psychological Science, 17(3), 236-242.

Roberti, J. W. (2004). A review of behavioral and biological correlates of sensation seeking. Journal of Research in Personality, 38, 256279. doi:10.1016/S0092-6566(03)00067-9

Schwartz, K. D., \& Fouts, G. T. (2003). Music preferences, personality style, and developmental issues of adolescents. Journal of Youth and Adolescence, 32(3), 205-221. doi: 10.1023/A:1022547520656

Singer, S. L., Levine, M., \& Jou, S. (1993). Heavy metal music preference, delinquent friends, social control and delinquency. Journal of Research in Crime and Delinquency, 30(3), 317-329. doi: 10.1177/0022427893030003004

Vasconcelos, T. C. (2004). Personalidade, valores e condutas antisociais de jovens. Dissertação de mestrado. Departamento de Psicologia, Universidade Federal da Paraíba, João Pessoa, PB.

Vasconcelos, T. C., Gouveia, V. V., Pimentel, C. E., \& Pessoa, V. S. (2008). Condutas desviantes e traços de personalidade: testagem de um modelo causal. Estudos de Psicologia, 25(1), 55-65. doi: http://dx.doi.org/10.1590/S0103-166X2008000100006

Weisskirch, R. S., \& Murphy, L. C. (2004). Friends, porn, and punk: Sensation seeking in personal relationships, internet activities, and music preference among college students. Adolescence, 39(154), 189-201.

Weston, R., \& Gore, P. A. (2006). A brief guide to structural equation modeling. The Counseling Psychologist, 34(5), 719-751. doi: $10.1177 / 0011000006286345$

Zuckerman, M. (1994). Behavioral expressions and biosocial bases of personality. New York: Cambridge University Press.

Zuckerman, M., Bushsbaum, M. S., \& Murphy, D. L. (1980). Sensation seeking and its biological correlates. Psychological Bulletin, 88(1), 187-214. 